\title{
Effects of physics beyond the standard model on the neutrino charge radius: an effective Lagrangian approach
}

\author{
H. Novales-Sánchez ${ }^{(a)}$, A. Rosado ${ }^{(b)}$, V. Santiago-Olán ${ }^{(b)}$, J. J. $\operatorname{Toscano}^{(a, c)}$ \\ ${ }^{(a)}$ Facultad de Ciencias Físico Matemáticas, Benemérita Universidad \\ Autónoma de Puebla, Apartado Postal 1152, Puebla, Pue., México. \\ ${ }^{(b)}$ Instituto de Física, Benemérita Universidad Autónoma de Puebla. Apdo. Postal J-48, C.P. 72570 Puebla, Pue., México. \\ ${ }^{(c)}$ Instituto de Física y Matemáticas, Universidad Michoacana de San Nicolás de Hidalgo, \\ Edificio C-3, Ciudad Universitaria, C.P. 58040, Morelia, Michoacán, México.
}

(Dated: September 22, 2021)

\begin{abstract}
In this work, we look for possible new physics effects on the electromagnetic charge and anapole form factors, $f_{Q}\left(q^{2}\right)$ and $f_{A}\left(q^{2}\right)$, for a massless Dirac neutrino, when these quantities are calculated in the context of an effective electroweak Yang-Mills theory, which induces the most general $S U_{L}(2)-$ invariant Lorentz tensor structure of nonrenormalizable type for the $W W \gamma$ vertex. It is found that in this context, besides the standard model contribution, the additional contribution to $f_{Q}\left(q^{2}\right)$ and $f_{A}\left(q^{2}\right)\left(f_{Q}^{O_{W}}\left(q^{2}\right)\right.$ and $f_{A}^{O_{W}}\left(q^{2}\right)$, respectively) are gauge independent and finite functions of $q^{2}$ after adopting a renormalization scheme. These form factors, $f_{Q}^{O_{W}}\left(q^{2}\right)$ and $f_{A}^{O_{W}}\left(q^{2}\right)$, get contribution at the one loop level only from the proper neutrino electromagnetic vertex. Besides, the relation $f_{Q}^{e f f}\left(q^{2}\right)=q^{2} f_{A}^{e f f}\left(q^{2}\right)\left(f_{Q}^{e f f}\left(q^{2}\right)=f_{Q}^{S M}\left(q^{2}\right)+f_{Q}^{O_{W}}\left(q^{2}\right), f_{A}^{e f f}\left(q^{2}\right)=f_{A}^{S M}\left(q^{2}\right)+f_{A}^{O_{W}}\left(q^{2}\right)\right)$ is still fulfilled and hence the relation $a_{\nu}^{\text {eff }}=\left\langle r_{\nu}^{2}\right\rangle^{\text {eff }} / 6\left(a_{\nu}^{\text {eff }}=a_{\nu}^{S M}+a_{\nu}^{O_{W}},\left\langle r_{\nu}^{2}\right\rangle^{e f f}=\left\langle r_{\nu}^{2}\right\rangle^{S M}+\left\langle r_{\nu}^{2}\right\rangle^{O_{W}}\right)$ is gotten, just as in the SM. Using the experimental constraint on the anomalous $W W \gamma$ vertex, a value for the additional contribution to the charge radius of $\left|\left\langle r_{\nu}^{2}\right\rangle^{O_{W}}\right| \lesssim 10^{-34} \mathrm{~cm}^{2}$ is obtained, which is one order of magnitude lower than the SM value.
\end{abstract}

PACS numbers: 13.15.+g, 13.40.Gp, 23.40.Bw, 25.30.Pt

\section{INTRODUCTION.}

Many authors have studied the neutrino charge radius (NCR) [1, 2, 3, 4, 5, 6, 7, 8, 9, 10, 11, 12, 13]. In 1972, Bardeen, Gastmans and Lautrup 2] showed in the frame of the SM and using the unitary gauge that the NCR is infinite and therefore it is not a physical quantity. Later, in the same year, S.Y. Lee [14] working in the unitary gauge considered the $\nu_{l} l^{\prime}$ scattering and defined the NCR including besides the usual terms, diagrams in which the photon is replaced by a neutral gauge boson $Z$. In this way he obtained a finite, although gauge dependent quantity [15]. One of the earliest analyses of the neutrino charge radius, in the context of the general one-loop electromagnetic form factor of a fermion in electroweak theories, was carried out in 1977 by Lee and Shrock [3]. These authors working in the context of the SM and using the linear $\mathrm{R}_{\xi}$ gauge showed explicitly that the NCR is not only infinite, but also gauge dependent. Lee and Shrock showed in their paper how a full calculation, including not just chargeradius terms, but also box diagrams (which could not be considered to be corrections to the neutrino electromagnetic vertex) combined together to yield a gauge-independent total amplitude. Hence, in order to look for a definition of a physical neutrino charge radius one has to consider other diagrams which contribute to the total amplitude of the physical process $\nu_{l} l^{\prime} \rightarrow \nu_{l} l^{\prime}$. The papers written by S.Y. Lee, and by B.W. Lee and R.E. Shrock inspired many works in which finite and gauge independent quantities, based on the NCR, were introduced by considering the $\nu_{l} l^{\prime}$ scattering $[15,16,17,18,19,20,21,22,23,24,25,26,27]$. We want to end this paragraph pointying out the following. Eventhough that it has been already shown that the neutrino charge radius is an infinite and gauge dependent quantity in the frame of the standard model (SM) when just the proper diagrams are taken into account, it is possible to define a physical neutrino charge radius by considering the $\nu_{l} l^{\prime}$ scattering, which becomes a finite and gauge independent quantity, independent of the lepton $l^{\prime}$ used to define it and also which only gets contribution from the proper neutrino electromagnetic vertex 18, 19, 20, 22]. Discussions on the experimental bounds on the NCR can be found, for example, in Refs. [28, 29, 30, 31, 32, 33]

On the other hand, it is well known that any fermion may develop an anapole moment $a$ [34]. In particular, the neutrino, even massless, may have an anapole moment $a_{\nu}$. Measurements of the solar neutrino flux at SuperKamiokande established that at least one neutrino is not massless [35]. Besides, from atmospheric and accelerator neutrino oscillations, we know that there is a non-vanishing mass difference [36]. From solar and reactor neutrino oscillations, we know that there is a different non-vanishing mass difference [37]. So, at least two neutrinos are not massless. The neutrino anapole moment (NAM) has been discussed in great detail in the literature 38, 39, 40, 41, 42, 43, 44, 45, 46]. Here, we only want to remark that in the frame of the standard model [47] it is satisfied the relation $a_{\nu}^{S M}=\left\langle r_{\nu}^{2}\right\rangle^{S M} / 6$ for a massless Dirac neutrino [40, 45]. The relation between the charge form factor and the anapole 
form factor for massless active (left-handed) neutrinos is not model-dependent. In fact, it is a consequence of having an effective vertex for the neutrino with the left-handed chirality projector.

In this work, we study possible new physics effects on the NCR in a model independent approach by using the effective Lagrangian technique [48, 49], which is an appropriate scheme to study those processes that are suppressed or forbidden in the SM. Motivated by the highly gauge dependent behavior of the charge and the anapole form factors, $f_{Q}\left(q^{2}\right)$ and $f_{A}\left(q^{2}\right)$, within the context of the SM, we will focus on those effects that could be induced by a Yang-Mills sector possessing a richer gauge structure than that of the dimension-four theory. To this end, we will consider an effective electroweak Yang-Mills sector that includes $S U_{L}(2)$-invariants of dimension higher than four. As we will see below, there is only one dimension-six $S U_{L}(2)$ invariant that induces the $W W \gamma$ vertex and contributes at the one-loop level to these form factors, $f_{Q}^{O_{W}}\left(q^{2}\right)$ and $f_{A}^{O_{W}}\left(q^{2}\right)$, respectively. Hence, we can write $f_{Q}^{e f f}\left(q^{2}\right)=f_{Q}^{S M}\left(q^{2}\right)+f_{Q}^{O_{W}}\left(q^{2}\right)$ and $f_{A}^{e f f}\left(q^{2}\right)=f_{A}^{S M}\left(q^{2}\right)+f_{A}^{O_{W}}\left(q^{2}\right)$, where $f_{Q}^{S M}\left(q^{2}\right)$ and $f_{A}^{S M}\left(q^{2}\right)$ are the standard model form factors. We will show that, as a consequence of the $S U_{L}(2)$ symmetry, the dimension-six $W W \gamma$ vertex gives a contribution which leads to manifest gauge independent expressions for the $f_{Q}^{O_{W}}\left(q^{2}\right)$ and $f_{A}^{O_{W}}\left(q^{2}\right)$ form factors. This result arises, in part, due to the fact that the $S U_{L}(2) \times U_{Y}(1)$ invariants of dimension higher than four are not affected by the gauge-fixing procedure used in the dimension-four theory. As a consequence, in the context of effective theories, fermionic form factors would be made of vertices that are not affected by the gauge-fixing procedure, which eventually would lead to gauge independent form factors [50]. Even more, we will show that it is possible to express these form factors as finite functions of $q^{2}$ by renormalizing them in the sense of effective field theories [51]. These form factors get contribution at the one loop level only from the proper neutrino electromagnetic vertex. Besides, for a massless neutrino and as a consequence of having an effective vertex for the neutrino with the left-handed chirality projector the relation $f_{Q}^{O_{W}}\left(q^{2}\right)=q^{2} f_{A}^{O_{W}}\left(q^{2}\right)$ is fulfilled. Hence, $f_{Q}^{e f f}\left(q^{2}\right)=q^{2} f_{A}^{e f f}\left(q^{2}\right)$ and therefore we get the relation $a_{\nu_{l}}^{\text {eff }}=\left\langle r_{\nu_{l}}^{2}\right\rangle^{\text {eff }} / 6$, as in the standard model.

This paper is organized as follows. In Sec III, the structure of the electromagnetic and anapole form factors of the neutrino in the context of the SM is briefly discussed, mainly to fix our notation. In SecIII, the calculation of the electromagnetic and anapole form factors of the neutrino in the context of an effective Yang-Mills electroweak theory is presented. The gauge independence of these form factors, as well as the possibility of introducing a renormalization scheme beyond the Dyson's sense, will be emphasized. Finally, in SecIV the conclusions are presented.

\section{THE ELECTROMAGNETIC STRUCTURE OF THE $\bar{\nu} \nu \gamma$ VERTEX IN THE SM}

In this section, we will present a schematic discussion on the main features of the charge and anapole form factors of a massless Dirac neutrino within the context of the SM [47]. We will take advantage to introduce the notation and conventions that will be used through the paper. For a massless left-handed neutrino the matrix element of the electromagnetic current can be expressed in terms of only one form factor $F\left(q^{2}\right)$ as

$$
\mathcal{M}_{\mu}=i e F\left(q^{2}\right) \bar{u}_{\nu}\left(p^{\prime}\right) \gamma_{\mu}\left(1-\gamma_{5}\right) u_{\nu}(p)
$$

For a massless neutrino, this expression can easily be rewritten as follows

$$
\mathcal{M}_{\mu}=i e \bar{u}_{\nu}\left(p^{\prime}\right)\left\{\gamma_{\mu} f_{Q}^{S M}\left(q^{2}\right)-\gamma_{\lambda} \gamma_{5}\left[g_{\mu}^{\lambda} q^{2}-q^{\lambda} q_{\mu}\right] f_{A}^{S M}\left(q^{2}\right)\right\} u_{\nu}(p)
$$

where [52 $f_{Q}^{S M}\left(q^{2}\right)=F\left(q^{2}\right)$ and $f_{A}^{S M}\left(q^{2}\right)=F\left(q^{2}\right) / q^{2}$ are the charge and anapole form factors of the neutrino, respectively. $f_{Q}^{S M}\left(q^{2}\right)$ satisfies the physical requirement:

$$
f_{Q}^{S M}(0)=0
$$

On the other hand, the NCR and the NAM are defined, respectively, by

$$
\left\langle r_{\nu}^{2}\right\rangle=\left.6 \frac{\partial f_{Q}^{S M}\left(q^{2}\right)}{\partial q^{2}}\right|_{q^{2}=0}=\left.6 \frac{\partial F\left(q^{2}\right)}{\partial q^{2}}\right|_{q^{2}=0}
$$

and

$$
a_{\nu}=f_{A}^{S M}(0)=\left.\frac{F\left(q^{2}\right)}{q^{2}}\right|_{q^{2}=0}=\left.\frac{\partial F\left(q^{2}\right)}{\partial q^{2}}\right|_{q^{2}=0}
$$




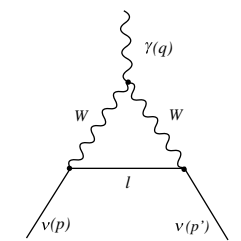

(a)

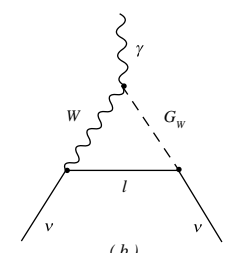

(b)
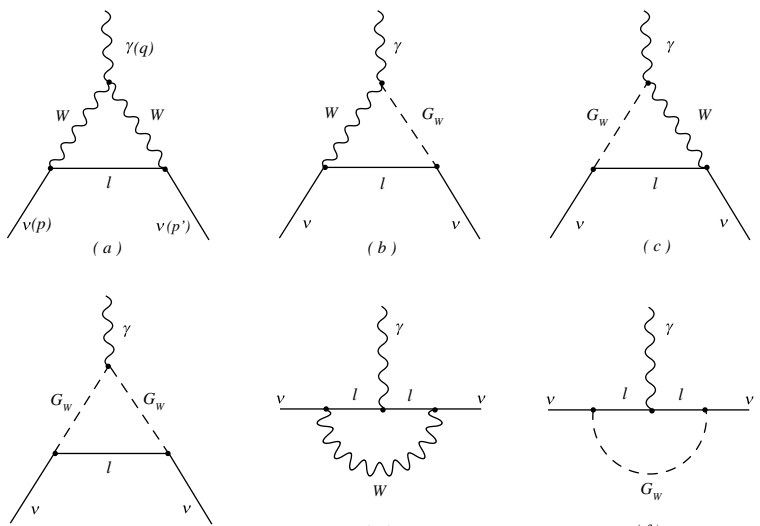

(d)

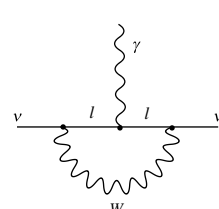

(e)

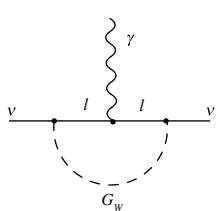

$(f)$

FIG. 1: Proper diagrams contributing to the neutrino charge radius in the standard model in the linear $R_{\xi}$ gauge.
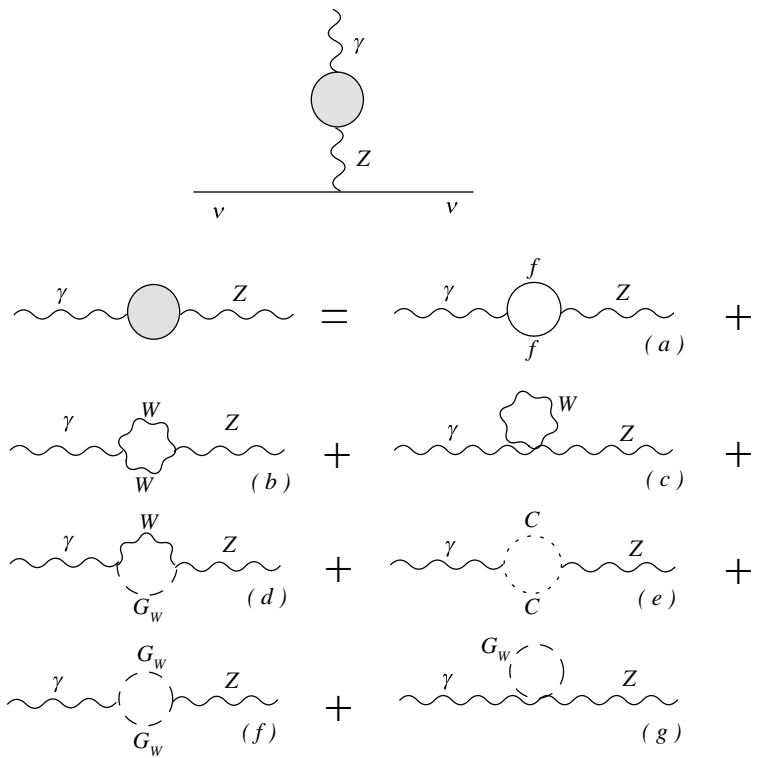

FIG. 2: Reducible diagrams contributing to the neutrino charge radius in the standard model in the linear $R_{\xi}$ gauge.

That is,

$$
a_{\nu}^{S M}=\left\langle r_{\nu}^{2}\right\rangle^{S M} / 6
$$

\section{ELECTROMAGNETIC STRUCTURE OF A DIRAC MASSLESS NEUTRINO IN AN EFFECTIVE YANG-MILLS THEORY}

We now turn to calculating the one-loop contribution of an anomalous (nonrenormalizable) $W W \gamma$ vertex to the charge and anapole form factors of the SM Dirac neutrino. Next, we will predict the corresponding charge radius by adopting an appropriate renormalization scheme.

\section{A. The most general gauge structure of the $W W \gamma$ vertex}

The gauge structure of the $W W \gamma$ vertex (and also of $W W Z$ ) has been the subject of important attention in the literature in diverse contexts. The one-loop radiative corrections to the renormalizable vertex have been calculated in 
the SM [53] and some of its extensions [54]. Its most general structure has been parametrized in a model independent manner using the effective Lagrangian approach [55, 56, 57] and used in countless phenomenological applications [58]. One effective electroweak Lagrangian can be constructed by adding to the dimension four Lagrangian all the $S U_{L}(2) \times$ $U_{Y}(1)$ invariants of dimension higher than four, which may respect or no discrete transformations, such as $P, T$, and $C$ or some combinations of them. The effective Lagrangian can be written as

$$
\mathcal{L}_{\text {eff }}=\mathcal{L}_{S M}+\sum_{n=5} \sum_{i=1}^{N} \frac{\alpha_{i}}{\Lambda^{n-4}} \mathcal{O}_{i},
$$

where $\mathcal{L}_{S M}$ represents the SM Lagrangian and $\mathcal{O}_{i}$ are $\left.S U_{(} 2\right) \times U_{Y}(1)$ invariants of dimension higher than four. $\Lambda$ represents the new physics scale and the $\alpha_{i}$ are unknown coefficients, which can be calculated once the fundamental theory is known. The $S U_{L}(2) \times U_{Y}(1)$ structure of some of the $\mathcal{O}_{i}$ operators depend on the mechanism responsible for the electroweak symmetry breaking. If this breaking occurs through the Higgs mechanism, the $S U_{L}(2) \times U_{Y}(1)$ symmetry is linearly realized through the Higgs doublet. If this is not the case, the electroweak symmetry is realized nonlinearly through the introduction of the matrix field $U=\exp \left(\sigma^{a} \phi^{a} / v\right)$ instead of the Higgs doublet. Here, the $\phi^{a}(x)$ fields represent the pseudo Goldstone bosons and $v$ is the Fermi scale. In this case, the effective Lagrangian parametrizes new physics that are the responsible for the electroweak symmetry breaking [59]. In this paper, we will focus on those type of effective interactions that are independent of the mechanism responsible for the electroweak symmetry breaking. As we will see below, this class of invariants induce the nonrenormalizable structure of the $W W \gamma$ vertex, which is dictated exclusively by the $S U_{L}(2)$ group.

From the known particles, the $W$ gauge boson, whose properties would exhaustively be studied at the next generation of linear colliders, is the one which possesses the richer collection of electromagnetic form factors, as it is charged and has the highest spin within the category of renormalizable theories. To our best knowledge, Gaemers and Gounaris [55] derived initially 9 form factors for the $W W \gamma$ vertex, but further on a careful analysis carried out by HagiwaraPeccei-Zeppenfeld-Hikasa [56] showed that only 7 of these quantities are independent indeed. Subsequent studies have confirmed these results [60]. These form factors define the charge, the magnetic and electric dipole moments, the magnetic and electric quadrupole moments, and the $\mathrm{CP}$-even and $\mathrm{CP}$-odd anapole moments of this particle. The $W W \gamma$ vertex is given by the following Lagrangian:

$$
\begin{aligned}
\mathcal{L}_{W W \gamma}= & i e\left[\left(W_{\mu \nu}^{-} W^{+\mu}-W_{\mu \nu}^{+} W^{-\mu}\right) A^{\nu}+F_{\mu \nu} W^{-\mu} W^{+\nu}\right. \\
& +2 \Delta \kappa_{\gamma} F_{\mu \nu} W^{-\mu} W^{+\nu}+\tilde{\kappa}_{\gamma} \tilde{F}_{\mu \nu} W^{-\mu} W^{+\nu}+\frac{\lambda_{\gamma}}{m_{W}^{2}} W_{\lambda \mu}^{-} W_{\nu}^{+\mu} F^{\nu \lambda}+\frac{\tilde{\lambda}_{\gamma}}{m_{W}^{2}} W_{\lambda \mu}^{-} W_{\nu}^{+\mu} \tilde{F}^{\nu \lambda} \\
& \left.-\frac{i \tilde{a}_{W}^{\gamma}}{m_{W}^{2}}\left(W_{\lambda \nu}^{+} W^{-\lambda}+W_{\nu \lambda}^{-} W^{+\lambda}\right) \partial_{\rho} F^{\rho \nu}-\frac{i a_{W}^{\gamma}}{m_{W}^{2}}\left(\tilde{W}_{\lambda \nu}^{+} W^{-\lambda}+\tilde{W}_{\lambda \nu}^{-} W^{+\lambda}\right) \partial_{\rho} F^{\rho \nu}\right],
\end{aligned}
$$

where $F_{\mu \nu}=\partial_{\mu} A_{\nu}-\partial_{\nu} A_{\mu}, \tilde{F}_{\mu \nu}=(1 / 2) \epsilon_{\mu \nu \alpha \beta} F^{\alpha \beta}$, etc. While the CP-even $\left(\Delta \kappa_{\gamma}, \lambda_{\gamma}\right)$ parameters define the magnetic dipole and electric quadrupole moments, the CP-odd ones $\left(\tilde{\kappa}_{\gamma}, \tilde{\lambda}_{\gamma}\right)$ determine the electric dipole and magnetic quadrupole moments of the $W$ gauge boson [56]. On the other hand, $a_{W}^{\gamma}$ and $\tilde{a}_{W}^{\gamma}$ represent the $\mathrm{CP}-$ even and $\mathrm{CP}-$ odd anapole moments of this particle. The only terms in the above equation which are explicitly invariant under the electromagnetic $U_{e}(1)$ gauge group are those associated with the form factors $\kappa_{\gamma}$ and $\tilde{\kappa}_{\gamma}$. However, the above interactions arise from a more fundamental Lagrangian which is invariant under the electroweak $S U_{L}(2) \times U_{Y}(1)$ gauge group. In the linear realization of the group [48, 49], the electroweak effective Lagrangian that induces $\mathcal{L}_{W W \gamma}$ can be written in terms of $S U_{L}(2) \times U_{Y}(1)$ invariants that comprise interactions of up to dimension eight as follows:

$$
\begin{aligned}
\mathcal{L}_{e f f}= & -\frac{1}{4} W_{\mu \nu}^{a} W^{a \mu \nu}+\frac{\alpha_{W B}}{\Lambda^{2}}\left(\Phi^{\dagger} W^{\mu \nu} \Phi\right) B_{\mu \nu}+\frac{\tilde{\alpha}_{W B}}{\Lambda^{2}}\left(\Phi^{\dagger} W^{\mu \nu} \Phi\right) \tilde{B}_{\mu \nu} \\
& +\frac{\alpha_{W}}{\Lambda^{2}} \frac{\epsilon_{a b c}}{3 !} W_{\lambda \mu}^{a} W_{\nu}^{b \mu} W^{c \nu \lambda}+\frac{\tilde{\alpha}_{W}}{\Lambda^{2}} \frac{\epsilon_{a b c}}{3 !} W_{\lambda \mu}^{a} W_{\nu}^{b \mu} \tilde{W}^{c \nu \lambda} \\
& +\frac{\alpha_{W D B}}{\Lambda^{4}}\left(i\left(\Phi^{\dagger} W_{\nu \lambda} D^{\lambda} \Phi\right)+\text { H.c. }\right) \partial_{\mu} B^{\mu \nu} \\
& +\frac{\tilde{\alpha}_{W D B}}{\Lambda^{4}}\left(i\left(\Phi^{\dagger} \tilde{W}_{\nu \lambda} D^{\lambda} \Phi\right)+\text { H.c. }\right) \partial_{\mu} B^{\mu \nu},
\end{aligned}
$$

where the dimension-four Yang-Mills term has been included. Here, $D^{\lambda}$ is the covariant derivative of the $S U_{L}(2) \times$ $U_{Y}(1)$ group and $W_{\mu \nu}^{a}\left(W_{\mu \nu}=\sigma^{a} W_{\mu \nu}^{a} / 2\right)$ and $B_{\mu \nu}$ are the respective field tensors. In addition, $\Phi$ is the Higgs doublet. The two dimension-eight operators were introduced in order to generate the anapole moments of the $W$ boson. They are made of the $S U_{L}(2) \times U_{Y}(1)$-invariant piece $\left(\Phi^{\dagger} W_{\nu \lambda} D^{\lambda} \Phi\right)$ 61] needed to generate the $W$ current. These operators, which have not been considered in the literature, can be eliminated using the equations of motion, as must be. As for the dimension-six invariants, their properties have widely been studied in the literature in diverse contexts [58]. 


\section{B. The anapole moment of the neutrino}

As already mentioned in Sec II a massless Dirac neutrino possess only two electromagnetic form factors, which are however not independent. These form factors are the charge, $f_{Q}\left(q^{2}\right)$, and the anapole, $f_{A}\left(q^{2}\right)$, which satisfy the relation $f_{Q}\left(q^{2}\right)=q^{2} f_{A}\left(q^{2}\right)$. The $W W \gamma$ vertex can contribute at the one-loop level to these form factors through the $\mathrm{CP}$-conserving structures. However, in this work we will focus on the contribution given by the $\mathrm{CP}-\mathrm{even} \lambda_{\gamma}$ component of this vertex, which is governed exclusively by the $S U_{L}(2)$ gauge group. A comprehensive analysis for the off-shell vertex $\bar{f} f V$, with $f$ an arbitrary fermion and $V=\gamma, Z$, will be presented elsewhere [50]. There are several good reasons to consider the contribution given by the $S U_{L}(2)$-invariant $\mathcal{O}_{W}=\left(\epsilon_{a b c} / 3\right.$ !) $W_{\lambda \mu}^{a} W^{b \mu}{ }_{\nu} W^{c \nu \lambda}$ to the neutrino form factors. In first place is its pure Yang-Mills nature, which leads to the most general structure for the gauge $W W \gamma$ vertex. This invariant constitutes therefore a good theoretical instrument to studying the gauge structure of electromagnetic form factors of elementary particles. Another important reason to use this operator is, as we will see below, that it leads to a NCR and NAM that are gauge independent in manifest way. Also, due its nonrenormalizable nature, $\mathcal{O}_{W}$ is necessarily generated at one-loop or higher orders in any renormalizable theory, which means that it maybe sensitive to new physics effects.

Using the notation given in Fig 3 , the vertex function, $\left(i e \lambda_{\gamma} / m_{W}^{2}\right) \Gamma_{\lambda \rho \mu}\left(k_{1}, k_{2}, k_{3}\right)$, for the $\frac{i e \lambda_{\gamma}}{m_{W}^{2}} W_{\lambda \mu}^{-} W^{+\mu} F^{\nu \lambda}$ interaction can be written as:

$$
\Gamma_{\lambda \rho \mu}\left(k_{1}, k_{2}, k_{3}\right)=\left(k_{3 \alpha} g_{\beta \rho}-k_{3 \beta} g_{\alpha \rho}\right)\left(k_{2}^{\beta} g_{\nu \lambda}-k_{2 \nu} \delta_{\lambda}^{\beta}\right)\left(k_{1}^{\nu} \delta_{\mu}^{\alpha}-k_{1}^{\alpha} \delta_{\mu}^{\nu}\right)
$$

which, as it is evident, satisfies the following simple Ward identities:

$$
\begin{gathered}
k_{1}^{\mu} \Gamma_{\lambda \rho \mu}\left(k_{1}, k_{2}, k_{3}\right)=0, \\
k_{2}^{\lambda} \Gamma_{\lambda \rho \mu}\left(k_{1}, k_{2}, k_{3}\right)=0, \\
k_{3}^{\rho} \Gamma_{\lambda \rho \mu}\left(k_{1}, k_{2}, k_{3}\right)=0 .
\end{gathered}
$$

We now turn to calculating the contribution of the above vertex to the NAM. Since the $\mathcal{O}_{W}$ operator is not affected by the gauge-fixing procedure of the dimension-four theory, there are no contributions from ghost fields. Also, there are not contributions from Goldstone bosons, as $\mathcal{O}_{W}$ does not depend on the mechanism responsible for the electroweak symmetry breaking. Accordingly, $\mathcal{O}_{W}$ only can contribute through the proper diagram of Fig $1(a)$ and the self-energy diagrams given in Figs $2(b)$ and $2(c)$. The latter one being induced by $\mathcal{O}_{W}$ through the quartic $W W Z \gamma$ vertex. From all the involved vertices, only the SM $W W \gamma$ one, which contributes through the diagram in Fig[2(b), depends on the gauge-fixing procedure. However, we have verified that due to the above Ward identities, this diagram gives a vanishing contribution. Also, we have encountered that there is no contribution from diagram in Fig. $2(c)$. So, the contribution to the NAM arises only through the proper diagram given in Fig $1(a)$, whose vertices are all independent of the gauge-fixing procedure. This means that the only gauge dependence of the NAM could arise through the $W$ propagator, which in the $R_{\xi}$ gauge is given by

$$
\Delta^{\mu \nu}=\frac{-i}{k^{2}-m_{W}^{2}}\left[g^{\mu \nu}-(\xi-1) \frac{k^{\mu} k^{\mu}}{k^{2}-\xi m_{W}^{2}}\right] .
$$

However, it is clear that due to the Ward identities given above, the longitudinal components of the $W$ propagators do not contribute to the proper diagram given in Fig 1 $(a)$. As a consequence, the amplitude for this diagram is manifestly gauge independent. This amplitude is given by

$$
\mathcal{M}_{\mu}^{O_{W}}=\frac{i g^{2} \lambda_{\gamma}}{2 m_{W}^{2}} \int \frac{d^{D} k}{(2 \pi)^{D}} \frac{P_{R} \gamma^{\rho} \not k \gamma^{\lambda} \Gamma_{\lambda \rho \mu}}{\left[k^{2}-m_{l}^{2}\right]\left[(k-p)^{2}-m_{W}^{2}\right]\left[\left(k-p^{\prime}\right)^{2}-m_{W}^{2}\right]},
$$

where $m_{l}$ is the mass of the charge lepton to which is associated the neutrino in consideration. Using the PassarinoVeltman [62] covariant decomposition, a direct calculation leads to the following expressions for the charge and anapole form factors:

$$
\begin{aligned}
& f_{Q}^{O_{W}}\left(q^{2}\right)=q^{2} f_{A}^{O_{W}}\left(q^{2}\right), \\
& f_{A}^{O_{W}}\left(q^{2}\right)=\frac{\alpha \lambda_{\gamma}}{8 \pi m_{W}^{2}} \frac{1}{1-x_{l}}\left[B_{0}(2)-x_{l} B_{0}(1)\right],
\end{aligned}
$$

where $B_{0}(1)=B_{0}\left(0, m_{l}^{2}, m_{W}^{2}\right)$ and $B_{0}(2)=B_{0}\left(q^{2}, m_{W}^{2}, m_{W}^{2}\right)$ are Passarino-Veltman two-point scalar functions. In addition, the dimensionless variable $x_{l}=m_{l}^{2} / m_{W}^{2}$ was introduced. The above expression was obtained after expressing a scalar $C_{0}$ three-point function as a combination of $B_{0}$ functions [63]. From the above expressions, it is clear that the $f_{Q}^{O_{W}}(0)=0$ condition is fulfilled. It is also evident that, although gauge independent, the anapole form factor is divergent. In the next subsection, the possibility of renormalizing this quantity within the context of effective field theories will be explored. 


\section{The neutrino charge radius}

The anapole form factor can be expressed in terms of elementary functions as follows:

$$
f_{A}^{O_{W}}\left(q^{2}\right)=\frac{\alpha \lambda_{\gamma}}{8 \pi m_{W}^{2}} \frac{1}{1-x_{l}}\left[\Delta\left(1-x_{l}\right)+2-x_{l}+\left(1-x_{l}\right) \log \left(\frac{\mu^{2}}{m_{W}^{2}}\right)-\frac{x_{l}^{2}}{1-x_{l}} \log \left(x_{l}\right)-g\left(x_{q}\right)\right],
$$

where $\mu$ is the dimensional regularization scale and the ultraviolet divergence is contained in:

$$
\Delta=\frac{2}{4-D}-\gamma_{E}+\log (4 \pi)
$$

with $\gamma_{E}$ the Euler's constant. In addition,

$$
g\left(x_{q}\right)= \begin{cases}2 \sqrt{\frac{4-x_{q}}{x_{q}}} \tan ^{-1}\left(\sqrt{\frac{x_{q}}{4-x_{q}}}\right), & \text { if } x_{q}<4 \\ \sqrt{\frac{x_{q}-4}{x_{q}}}\left[\log \left(\frac{1+\sqrt{\frac{x_{q}-4}{x_{q}}}}{1-\sqrt{\frac{x_{q}-4}{x_{q}}}}\right)-i \pi\right], & \text { if } x_{q}>4\end{cases}
$$

where $x_{q}=q^{2} / m_{W}^{2}$. Following Refs. [49, 51], the divergent term in this amplitude can be absorbed by renormalizing the coefficients of $\mathcal{L}_{\text {eff }}$ since it already contains all the invariants allowed by the SM symmetry. The invariant needed to absorb the divergence is

$$
\frac{\alpha_{A}}{\Lambda^{2}}\left(\bar{L} \gamma_{\nu} L\right) \partial_{\mu} B^{\mu \nu}
$$

where $L$ is the usual lepton doublet and $B^{\mu \nu}$ is the gauge tensor associated with the $U_{Y}(1)$ group. In this way, the divergence can be absorbed by the $\alpha_{A}$ parameter of the effective Lagrangian. Here, we want to point out the following. The divergency of Eq. (18) needs a counterterm in the effective neutrino-photon vertex to be renormalized. The invariant of Eq. (21) absorbs this divergence. However, after renormalization, the contribution of the counterterm is not zero, but a finite value. As a consequence, the final result is, in fact, the sum of two finite contributions: one explicit depending on the effective $W W \gamma$ coupling and a second one coming from the counterterm proportional to $\alpha_{A}$. The result quoted in this paper originates from the first contribution. Using the $\overline{M S}$ renormalization scheme with $\mu=\Lambda$, the renormalized anapole form factor can be written as follows:

$$
f_{A}^{O_{W}}\left(q^{2}\right)=\frac{\alpha \lambda_{\gamma}}{8 \pi m_{W}^{2}} \frac{f\left(x_{q}\right)}{1-x_{l}}
$$

where

$$
f\left(x_{q}\right)=2-x_{l}+\left(1-x_{l}\right) \log \left(\frac{\Lambda^{2}}{m_{W}^{2}}\right)-\frac{x_{l}^{2}}{1-x_{l}} \log \left(x_{l}\right)-g\left(x_{q}\right) .
$$

The anapole moment is the on-shell quantity $a_{\nu}=f_{A}\left(q^{2}=0\right)$. In this limit, the loop function $f\left(q^{2}\right)$ takes the way

$$
\begin{aligned}
f(0) & =\left(1-x_{l}\right)\left[2 \log \left(\frac{\Lambda}{m_{W}}\right)+1\right]-\frac{x_{l}^{2}}{1-x_{l}} \log \left(x_{l}\right), \\
& \approx 2 \log \left(\frac{\Lambda}{m_{W}}\right)+1
\end{aligned}
$$

which leads to the following expression for the additional contribution in the frame of an effective theory to the neutrino charge radius

$$
\begin{aligned}
\left\langle r_{\nu}^{2}\right\rangle^{O_{W}} & =\frac{3 \alpha \lambda_{\gamma}}{4 \pi m_{W}^{2}}\left[2 \log \left(\frac{\Lambda}{m_{W}}\right)+1\right], \\
& =0.95 \times 10^{-34} \mathrm{~cm}^{2} \lambda_{\gamma}\left[2 \log \left(\frac{\Lambda}{m_{W}}\right)+1\right] .
\end{aligned}
$$

We now turn to discussing our results. In order to make predictions, we need to assume some value for the $\lambda_{\gamma}$ parameter and the new physics scale $\Lambda$. We will use the most recent experimental limit on $\lambda_{\gamma}$ obtained by the D0 


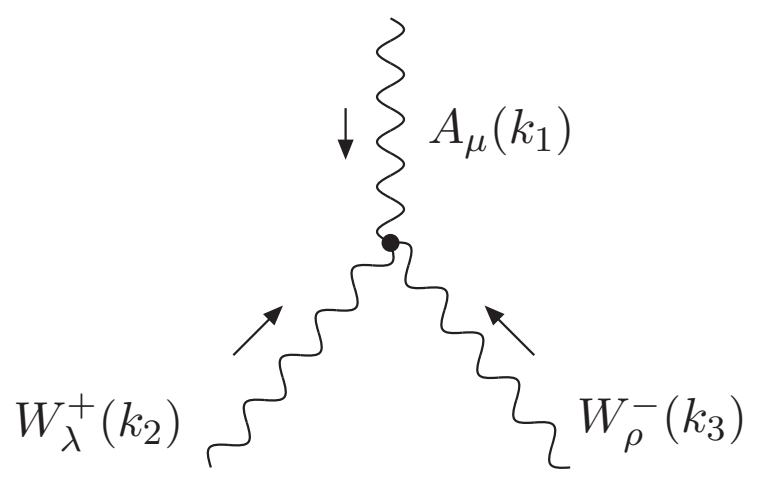

FIG. 3: The trilinear $W W \gamma$ vertex.

Collaboration [64], namely, $-0.29<\lambda_{\gamma}<0.30$ for $\Lambda=2.0 \mathrm{TeV}$. More recently, this Collaboration limited the trilinear $W W Z$ vertex to $-0.17<\lambda_{Z}<0.21$ at the $95 \%$ C.L. and for the same value of $\Lambda$ [65]. We will make predictions assuming that $\left|\lambda_{\gamma}\right| \lesssim 0.3$ and $\Lambda=2.0 \mathrm{TeV}$. Using these values, we obtain the following additional contribution to the neutrino charge radius:

$$
\left|\left\langle r_{\nu}^{2}\right\rangle^{O_{W}}\right| \lesssim 2 \times 10^{-34} \mathrm{~cm}^{2} .
$$

It is interesting to compare our result with the theoretical one obtained, within the frame of the SM and by using the Pinch Technique [66], in Ref. [18]. In this reference, it was derived that $\left\langle r_{\nu_{l}}^{2}\right\rangle^{S M}=4.1,2.4,1.5 \times 10^{-33} \mathrm{~cm}^{2}$ for $l=e, \mu, \tau$, respectively. Hence we can conclude that our result is about one order of magnitude lower than the results derived in Ref. [18]. On the other hand, our result is of the same order of magnitude than the new physics contribution derived in the context of the minimal supersymmetric standard model 67].

Finally, it is worthwhile to compare our result for the additional contribution in the context of an effective theory to the neutrino charge radius with some experimental bounds existing in the literature. The upper bound $\left\langle r_{\nu}^{2}\right\rangle^{\exp <}$ $7 \times 10^{-33} \mathrm{~cm}^{2}$ was derived from primordial nucleosynthesis [28]. In Ref. 29], the bound $\left\langle r_{\nu}^{2}\right\rangle^{e x p}<2 \times 10^{-33} \mathrm{~cm}^{2}$ for the charge radius of a right-handed neutrino was derived using astrophysical information obtained from observational data on the SN 1987A. The bound $-2.74 \times 10^{-32}<\left\langle r_{\nu}^{2}\right\rangle^{\exp }<4.88 \times 10^{-33} \mathrm{~cm}^{2}$ was derived in Ref. [30] from neutralcurrent neutrino reaction. In Ref. [31] the upper limit $\left\langle r_{\nu}^{2}\right\rangle\left\langle 2 \times 10^{-32} \mathrm{~cm}^{2}\right.$ was derived from Kamiokande II and Chlorine solar experiments. From the above considerations, we can see that in general terms our result for $\left|\left\langle r_{\nu}^{2}\right\rangle^{O_{W}}\right|$ is about one order of magnitude lower that the best bounds derived so far.

\section{CONCLUSIONS}

Our aim in this paper has been to present the calculation of the additional contribution, in the frame of an effective Yang-Mills theory, to the charge and the anapole form factors, $f_{Q}^{O_{W}}\left(q^{2}\right)$ and $f_{A}^{O_{W}}\left(q^{2}\right)$, for a massless Dirac neutrino. These form factors get contribution at the one loop level only from the proper neutrino electromagnetic vertex. We showed that this vertex function is independent of the gauge-fixing parameter and ultraviolet finite, as it can be renormalized within the framework of the effective theory. We also showed that the relation $f_{Q}^{O_{W}}\left(q^{2}\right)=q^{2} f_{A}^{O_{W}}\left(q^{2}\right)$ is fulfilled and hence we get the relation $a_{\nu}^{O_{W}}=\left\langle r_{\nu}^{2}\right\rangle^{O_{W}} / 6$. Therefore, $a_{\nu}^{e f f}=\left\langle r_{\nu}^{2}\right\rangle^{e f f} / 6$ as in the standard model. This well-known relation between the charge form factor and the anapole form factor for massless active (left-handed) neutrinos does not depend on an specific model. This relation is a consequence of having an effective vertex for the neutrino with the left-handed chirality projector.

An interesting point, long discussed in the literature, is the gauge independence and non-divergence of the neutrino charge radius. In the SM, this issue is now clarified with a definition of this quantity with all the required properties, which is furthermore gauge independent and finite. In the model discussed by the present paper, one should again treat these points with care, particularly in the context of effective theories. The question of gauge independence is solved favorably because only the transverse part of the W-propagator appears. However, the divergency of Eq. (18) needs a counterterm in the effective neutrino-photon vertex to be renormalized. As it was stated in subsection III.C, the invariant of Eq. (21) absorbs this divergence. However, after renormalization, the contribution of the counterterm is not zero, but a finite value. As a consequence, the final result is the sum of two finite contributions: one explicit depending on the effective $W W \gamma$ coupling and a second one coming from the counterterm proportional to $\alpha_{A}$. The result quoted in this work originates from the first contribution. 
Finally, we obtain $\left\langle r_{\nu}^{2}\right\rangle^{O_{W}} \approx \frac{3}{4}\left(\alpha \lambda_{\gamma} / m_{W}^{2}\right)\left[2 \log \left(\left(\Lambda^{2} / M_{W}^{2}\right)+1\right]\right.$. Using the recent D0 Collaboration constraint on the $\lambda_{\gamma}$ parameter, we estimate the value $\left|\left\langle r_{\nu}^{2}\right\rangle^{O_{W}}\right| \lesssim 2 \times 10^{-34} \mathrm{~cm}^{2}$, which is of the order of magnitude that may be expected in theories beyond the SM, as supersymmetry, and about one order of magnitude lower than the current bounds.

\section{ACKNOWLEDGMENTS}

This work was supported in part by the Consejo Nacional de Ciencia y Tecnología (CONACyT) and Sistema Nacional de Investigadores (SNI) de México. A.R. would like to thank Prof. R.E. Shrock for valuable comments.

[1] J. Bernstein and T. D. Lee, Phys. Rev. Lett. 11, 512 (1963).

[2] W. A. Bardeen, R. Gastmans, and B. Lautrup, Nucl. Phys. B 46, 319 (1972).

[3] B. W. Lee and R. E. Shrock, Phys. Rev. D 16, 1444 (1977).

[4] J. E. Kim, V. S. Mathur, and S. Okubo, Phys. Rev. D 9, 3050 (1974).

[5] J. E. Kim, Phys. Rev. D 14, 3000 (1976).

[6] D. Y. Bardin and O. A. Mogilevsky, Lett. Nuovo Cim. 9, 549 (1974).

[7] G. J. Aubrecht and K. Tanaka, Phys. Rev. D 10, 3522 (1974).

[8] N. M. Monyonko and J. H. Reid, Prog. Theor. Phys. 73, 734 (1985).

[9] P. Vogel and J. Engel, Phys. Rev. D 39, 3378 (1989).

[10] K. L. Ng, Nuovo Cim. A 107, 431 (1994).

[11] P. Minkowski, P. Schupp and J. Trampetic, Eur. Phys. J. C 37, 123 (2004) arXiv:hep-th/0302175.

[12] M. Dvornikov and A. Studenikin, Phys. Rev. D 69, 073001 (2004) arXiv:hep-ph/0305206.

[13] M. S. Dvornikov and A. I. Studenikin, J. Exp. Theor. Phys. 99, 254 (2004) arXiv:hep-ph/0411085.

[14] S. Y. Lee, Phys. Rev. D 6, 1701 (1972).

[15] J. L. Lucio, A. Rosado and A. Zepeda, Phys. Rev. D 29, 1539 (1984); Phys. Rev. D 31,1091 (1985).

[16] G. Degrassi, W. J. Marciano and A. Sirlin, Phys. Rev. D 39, 287 (1989).

[17] M. J. Musolf and B. R. Holstein, Phys. Rev. D 43, 2956 (1991).

[18] J. Bernabeu, L. G. Cabral-Rosetti, J. Papavassiliou and J. Vidal, Phys. Rev. D 62, 113012 (2000) arXiv:hep-ph/0008114.

[19] J. Bernabeu, J. Papavassiliou and J. Vidal, Nucl. Phys. B 680, 450 (2004) arXiv:hep-ph/0210055.

[20] J. Bernabeu, J. Papavassiliou and J. Vidal, Phys. Rev. Lett. 89, 101802 (2002) [Erratum-ibid. 89, 229902 (2002)] arXiv:hep-ph/0206015.

[21] E. Nardi, AIP Conf. Proc. 670, 118 (2003) arXiv:hep-ph/0212266].

[22] J. Papavassiliou, J. Bernabeu and J. Vidal, Nucl. Phys. Proc. Suppl. 114, 197 (2003) arXiv:hep-ph/0210312.

[23] M. Hirsch, E. Nardi and D. Restrepo, Phys. Rev. D 67, 033005 (2003) arXiv:hep-ph/0210137.

[24] K. Fujikawa and R. Shrock, Phys. Rev. D 69, 013007 (2004) arXiv:hep-ph/0309329.

[25] J. Papavassiliou, J. Bernabeu, D. Binosi and J. Vidal, Eur. Phys. J. C 33, S865 (2004) arXiv:hep-ph/0310028.

[26] J. Bernabeu, J. Papavassiliou and D. Binosi, Nucl. Phys. B 716, 352 (2005) arXiv:hep-ph/0405288.

[27] J. Papavassiliou, J. Bernabeu and M. Passera, PoS HEP2005, 192 (2006) arXiv:hep-ph/0512029.

[28] J. A. Grifols and E. Masso, Mod. Phys. Lett. A 2, 205 (1987).

[29] J. A. Grifols and E. Masso, Phys. Rev. D 40, 3819 (1989).

[30] R. C. Allen et al., Phys. Rev. D 43, 1 (1991).

[31] A. M. Mourao, J. Pulido and J. P. Ralston, Phys. Lett. B 285, 364 (1992) [Erratum-ibid. B 288, 421 (1992)].

[32] P. Salati, Astropart. Phys. 2, 269 (1994).

[33] J. Barranco, O. G. Miranda and T. I. Rashba, arXiv:0707.4319 [hep-ph].

[34] Ya. B. Zeldovich, Soviet Phys. JETP, 33, 1531 (1957); Ya. B. Zeldovich and A. M. Perelomov, Soviet Phys. JETP, 12, 777 (1961); R. E. Marshak, Riazuddin and C. P. Ryan: Theory of weak interactions in particle physics: Wiley-Interscience.

[35] Y. Fukuda et al. [Super-Kamiokande Collaboration], Phys. Rev. Lett. 81, 1158 (1998) [Erratum-ibid. 81, 4279 (1998)] arXiv:hep-ex/9805021.

[36] D. G. Michael et al. [MINOS Collaboration], Phys. Rev. Lett. 97, 191801 (2006) arXiv:hep-ex/0607088.

[37] S. Abe et al. [KamLAND Collaboration], Phys. Rev. Lett. 100, 221803 (2008) arXiv:0801.4589 [hep-ex]].

[38] S. M. Apenko and Yu E. Lozovik, J. Phys. B 15, 257 (1982).

[39] A. Barroso, F. Boudjema, J. Cole and N. Dombey, Z. Phys. C 28, 149 (1985).

[40] A. Rosado, Rev. Mex. Fis. 47, 132 (2002).

[41] M. Abak and C. Aydin, Europhys. Lett. 4, 881 (1987).

[42] A. Góngora-T and Robin G. Stuart, Z. Phys. C 55, 101 (1992).

[43] H. Czyz, K. Kolodziej, M. Zralek and P. Christova, Can. J. Phys. 66, 132 (1988).

[44] H. Czyz and M. Zralek, Can. J. Phys. 66, 384 (1988).

[45] A. Rosado, Phys. Rev. D 61, 013001 (2000).

[46] L. G. Cabral-Rosetti, M. Moreno and A. Rosado, arXiv:hep-ph/0206083.

[47] S. Weinberg, Phys. Rev. Lett. 19 (1967) 1264; A. Salam: Proc. 8th Nobel Symposium, S. 367 (N. Svartholm, Ed., Stockholm 1968); S.L. Glashow, J. Illiopoulos and L. Maiani, Phys. Rev. D 2 (1970) 1285 
[48] W. Buchmuller and D. Wyler, Nucl. Phys. B 268, 621 (1986).

[49] J. Wudka, Int. J. Mod. Phys. A 9, 2301 (1994).

[50] H. Novales-Sánchez, V. Santiago-Olán, and J. J. Toscano, work in progress.

[51] S. Weinberg, Physica A 96, 327 (1979); J. Gasser and L. Leutwyler, Nucl. Phys. B 250, 465 (1985).

[52] Theory of weak interactions in particle physics: R. E. Marshak, Riazuddin and C. P. Ryan, Willey-Interscience 1998, p. 231.

[53] W. A. Bardeen, R. Gastmans, and B. Lautrup, Nucl. Phys. B 46, 319 (1972); see also E. N. Argyres et al., ibid. 391, 23 (1993); G. Couture, J. N. Ng, Z. Phys. C 35, 65 (1987).

[54] G. Couture, J. N. Ng, J. L. Hewett, and T. G. Rizzo, Phys. Rev. 36, 859 (1987); C. L. Bilachak, R. Gastmans, and A. van Proeyen, Nucl Phys. B 273, 46 (1986); G. Couture, J. N. Ng, J. L. Hewett, and T. G. Rizzo, Phys. Rev. D 38, 860 (1988); A. B. Lahanas and V. C. Spanos, Phys. Lett. B 334, 378 (1994); T. M. Aliyev, ibid. 155, 364 (1985); A. Arhrib, J. L. Kneur, and G. Moultaka, ibid. 376, 127 (1996); N. K. Sharma, P. Saxena, S. Singh, A. K. Nagawat, and R. S. Sahu, Phys. Rev. D 56, 4152 (1997); T. G. Rizzo and M. A. Samuel, Phys. Rev. D 35, 403 (1987); A. J. Davies, G. C. Joshi, and R. R. Volkas, ibid. 42, 3226 (1990); F. Larios, J. A. Leyva, and R. Martínez, Phys. Rev. D 53, 6686 (1996); G. Tavares-Velasco and J. J. Toscano, Phys. Rev. D 65, 013005 (2001); ibid. 69, 017701 (2004); J. L. García-Luna, G. Tavares-Velasco, and J. J. Toscano, Phys. Rev. D 69, 093005 (2004); J. Hernández-Sánchez, C. G. Honorato, F. Procopio, G. Tavares-Velasco, and J. J. Toscano, Phys. Rev. D 75, 073017 (2007).

[55] K. J. F. Gaemers and G. J. Gounaris, Z. Phys. C 1, 259 (1979).

[56] K. Hagiwara, R. D. Peccei, D. Zeppenfeld, and K. Hikasa, Nucl. Phys. B 282, 253 (1987).

[57] H. Novales-Sánchez and J. J. Toscano, Effective Lagrangian approach to fermion electric dipole moments induced by a $C P$-violating $W W \gamma$ vertex, to appear in Phys. Rev. D, arXiv:0712.2008 [hep-ph].

[58] See for instance, A. De Rújula, M. B. Gavela, O. Pene, and F. J. Vegas, Nucl. Phys. 357, 311 (1991); A. De Rújula, M. B. Gavela, P. Hernández, and E. Masso, Nucl. Phys. B 384, 3 (1992); C. Arzt, M. B. Einhorn, and J. Wudka, Phys. Rev. D 49, 1370 (1994); T. Inami, C. S. Lim, B. Takeuchi, and M. Tanabashi, Phys. Lett. B 381, 458 (1996); J. Ellison and J. Wudka, Ann. Rev. Nucl. Part. Sci. 48, 33 (1998). See also, J. Ellison and J. Wudka, Ann. Rev. Nucl. Part. Sci. 48, 33 (1998).

[59] See for instance, María José Herrero, hep-ph/9601286 T. Appelquist and G-H. Wu, Phys. Rev. D 51, 240 (1995).

[60] A comprehensive discussion on anapole moment of Majorana particles is given in: F. Boudjema and C. Hamzaoui, Phys. Rev. D 43, 3748 (1991). See also, F. Boudjema C. Hamzaoui, V. Rahal, and H. C. Ren, Phys. Rev. Lett. 62, 852 (1989).

[61] This $S U_{L}(2) \times U_{Y}(1)$-invariant structure was used to construct the operators that generate the trilinear neutral gauge boson couplings $Z Z Z, Z Z \gamma$, and $Z \gamma \gamma$ : F. Larios, M. A. Pérez, G. Tavares-Velasco, and J. J. Toscano, Phys. Rev. D 63, 113014 (2001).

[62] G. Passarino and M. J. G. Veltman, Nucl. Phys. B 160, 151 (1979).

[63] G. Devaraj and R. G. Stuart, Nucl. Phys. B 519, 483 (1998).

[64] V. M. Abazov et al. (D0 Collaboration), Phys. Rev. D 74, 057101 (2006).

[65] V. M. Abazov et al. (D0 Collaboration), arXiv:0709.2917 [hep-ph].

[66] J. M. Cornwall, Phys. Rev. D 26, 1453 (1982); J. M. Cornwall and J. Papavassiliou, Phys. Rev. D 40, 3474 (1989); J. Papavassiliou, Phys. Rev. D 41, 3179 (1990).

[67] K. L. Ng, Z. Phys. C 55, 145 (1992). 